\title{
Pentapeptide overlapping between human immunodeficiency viruses and Home sapiens proteomes is higher than $90 \%$
}

\section{Darja Kanduc}

\author{
Address: University of Bari, Bari, Italy \\ from Frontiers of Retrovirology: Complex retroviruses, retroelements and their hosts \\ Montpellier, France. 21-23 September 2009 \\ Published: 24 September 2009 \\ Retrovirology 2009, 6(Suppl 2):P44 doi:10.1 I86/1742-4690-6-S2-P44
}

This abstract is available from: http://www.retrovirology.com/content/6/S2/P44

(C) 2009 Kanduc; licensee BioMed Central Ltd.

We used sequence-to-sequence peptide matching to analyze the similarity level of the HIV-1, HIV-2 and HTLV polyprotein sequences to the human proteome. The following results were obtained: 1$)$ pentapeptides from the viral polyproteins are widely, repeatedly and intensively represented in a large number of human proteins; 2) high level of similarity of viral versus human proteomes is still present even using esa- or eptapeptide motifs as probes for sequence identity scanning; 3 ) a relatively limited number of viral pentameric fragments (about $10 \%$ or less) are unique to the viruses with no similarity to the human host; 4 ) the distribution of the zero similarity pentapeptides is not random in the three main immunodeficiency virus strains, with a maximum clustering in the HIV-2 p160 aa 1007-1019 sequence. The data are important in relation to the role of molecular mimicry in the genesis and diagnosis of immunodeficiency diseases, and the use of low similarity amino acid sequences as a peptidome platform for anti-HIV vaccine constructs exempt from harmful collateral cross-reactions. 